\title{
La dispersion comme ressource
}

Emmanuel Ma Mung

\section{(2) OpenEdition}

1 Journals

\section{Édition électronique}

URL : https://journals.openedition.org/conflits/225

DOI : $10.4000 /$ conflits. 225

ISSN : $1777-5345$

Éditeur :

CECLS - Centre d'études sur les conflits - Liberté et sécurité, L'Harmattan

Édition imprimée

Date de publication : 15 mai 1999

ISSN : 1157-996X

Référence électronique

Emmanuel Ma Mung, "La dispersion comme ressource », Cultures \& Conflits [En ligne], 33-34 | printemps-été 1999, mis en ligne le 16 mars 2006, consulté le 21 septembre 2021. URL : http:// journals.openedition.org/conflits/225 ; DOI : https://doi.org/10.4000/conflits.225

Ce document a été généré automatiquement le 21 septembre 2021.

Creative Commons License 


\title{
La dispersion comme ressource
}

\author{
Emmanuel Ma Mung
}

1 De quelle manière la dispersion géographique d'un corps social peut-elle être utilisée comme une "ressource spatiale " ? Cette idée de ressource spatiale est une notion qui reste à élaborer car, à notre connaissance, elle n'a pas été utilisée en tant que telle. Elle serait pourtant utile pour comprendre la façon dont s'organisent, à l'initiative de migrants internationaux, certaines circulations de personnes et de valeurs matérielles et immatérielles dans le monde contemporain. Cet article est une contribution à l'élaboration de cette notion. Tant qu'elle est pure et simple dispersion, atomisation de sujets n'entretenant pas de relations entre eux, la dispersion n'est pas utilisable. Elle peut même être ressentie comme négative. C'est ainsi que peut être vécue la migration : l'individu isolé, loin du pays d'origine, vit sa situation comme un exil, il éprouve une souffrance qui se traduit en nostalgie, une abondante littérature décrit cette situation. Elle devient " positive » dès lors qu'elle est valorisée et revendiquée par les sujets, à l'instar de ce que l'on peut observer dans certains entretiens avec des migrants où le fait d'avoir des parents ou des connaissances dans d'autres pays est présenté comme un objet de satisfaction et de fierté, proportionnelles au nombre de pays et de relations. Elle peut alors être utilisée pour faire des choses que l'on ne pourrait pas réaliser autrement. Lorsqu'une certaine disposition dans l'espace permet des réalisations difficiles à exécuter dans un autre situation, cette disposition spatiale devient une ressource - et l'on pourrait ajouter en jouant un peu des mots qu'elle devient une disposition sociale puisqu'elle va être l'argument justifiant une certaine façon de se concevoir comme corps social. Une ressource spatiale est alors une disposition dans l'espace susceptible d'être utilisée par les sujets à leur profit. Comment devient-il possible que la dispersion se transforme en une ressource? La diaspora chinoise nous en fournit une illustration ${ }^{1}$. Plusieurs processus étroitement imbriqués permettent cette transformation; ils prennent leur origine dans le rapport spécial que la diaspora entretient au territoire et dans l'effort qui est fait au sein de la diaspora pour penser l'unité d'un corps dispersé2. L'un tient en la fabrication d'une continuité généalogique à travers la création d'une mémoire/ histoire propre à la diaspora. Les termes de fabrication et de création sont utilisés pour bien souligner le caractère d'artefact de ces phénomènes et le fait qu'il n'y a pas d'histoire ou de disposition spatiale " naturelles » 
de ce groupe. Un autre processus concerne la façon selon laquelle cette continuité généalogique est transformée en contiguïté géographique. Un autre enfin consiste en la manière dont le rapport au territoire au sens classique d'espace circonscrit par la présence pérenne d'une population est remplacé par un sentiment d'extraterritorialité. Après avoir décrit ces processus nous verrons comment des ressources spatiales sont mobilisées.

2 Continuité généalogique Les Chinois d'outre-mer constituent une diaspora comprise au double sens de disposition particulière d'une population dans l'espace caractérisée par la multipolarisation de la migration et par l'interpolarité des relations, et, de population ayant conscience de cette disposition ${ }^{3}$. Nous en venons ainsi aux caractères morphologiques qui définissent, à notre avis, une diaspora: la multipolarité de la migration qui correspond à la diaspora au sens étymologique (du grec speiro, semer) et l'interpolarité des relations c'est à dire l'existence de relations entre les différents pôles de la diaspora. La diaspora chinoise est constituée de communautés locales reliées entre elles par des relations de diverse nature, flux de personnes, d'informations, de richesses etc. Insérées économiquement aux Etats-nations (par exemple, la France), ces communautés sont confrontées à des modèles d'intégration très différents selon les pays. Selon les cas leur présence est tolérée ou problématique, et le sentiment de la menace de l'expulsion est souvent présent quand ce n'est pas leur intégrité physique qui est menacée (voir en Indonésie récemment). Bien qu'intégrées aux nations ${ }^{4}$, elles fabriquent néanmoins leur intégration à la diaspora à travers la constitution d'une mémoire et d'une histoire collectives singulières dans lesquelles s'élaborent des généalogies et des filiations diasporiques entre les communautés locales. Cette mémoire/histoire leur permet d'articuler le sens de leur présence en un lieu à celui de la présence de la diaspora à laquelle ils donnent ainsi une tangibilité. Si cette présence de la diaspora devient ainsi recevable par les communautés locales, leur présence en un lieu devient en même temps redevable de la diaspora puisqu'elle va s'expliquer pour une part, à leurs yeux, par leur appartenance à celle-ci.

3 Au cours de la migration se développe une identité fondée sur le sentiment de partager une origine commune, réelle ou supposée, laquelle peut s'exprimer à travers des échelles différentes qui ne sont pas exclusives l'une de l'autre (même groupe ethnique, et/ou sur un plan géographique, même village ou quartier, même région, pays, voire même continent d'origine). Ce sentiment d'appartenance à un même groupe ayant une origine commune constitue une identité collective de nature ethnique ${ }^{5}$, que celle-ci s'appuie pour tout ou en partie sur une identité ethnique préalablement existante ou qu'elle soit une construction circonstancielle liée au mouvement migratoire. Cette origine peut donc être simplement géographique ou ethno-culturelle et généalogique, mais les deux se recouvrent souvent au cours de la migration, de sorte que le fait d'être d'un même endroit induit une sorte de généalogie : on procède du même lieu. C'est dire qu'en dernier ressort la forme généalogique domine à travers une sorte d'anthropomorphisation de la trajectoire géographique : venir d'un même endroit c'est au fond sortir de la même matrice. Cette identité sociale, en ce qu'elle s'édifie sur une supposée origine commune, est orientée vers le passé et vers la construction d'une mémoire collective. La constitution de cette mémoire peut prendre une forme savante (le rôle des intellectuels dans ce cas est prépondérant à travers des travaux historiques dont l'essentiel est la reconstitution de l'histoire du groupe ou des travaux sociologiques qui examinent, en même temps qu'ils la renforcent, l'identité collective, 
ou encore des productions artistiques...) ou courante (pratiques alimentaires, habillement, décoration intérieure, pratiques religieuses, langue...) Cette construction/ reconstitution d'une mémoire commune est essentielle aussi bien dans sa dimension savante que courante pour argumenter et se convaincre d'une origine commune. À cet égard, l'exemple de la diaspora chinoise est parlant : il existe, parfois depuis plusieurs décennies, des musées consacrés aux Chinois d'outre-mer dans diverses villes d'installation telles que San Francisco (haut lieu fortement symbolique pour la migration chinoise) ou dans des villes de départ telles que Xiamen (province du Fujian, une des principales aires d'origine); ces musées consistent en l'exposition d'objets témoins de la migration (photographies, passeports, visas, correspondances, coupures de presse, vêtements d'époque...). En France même, il y a eu plusieurs tentatives de création d'un musée consacré à la présence chinoise dans ce pays. L'immigration en nombre important de personnes d'origine chinoise y est pourtant récente, si l'on excepte la venue durant la Grande Guerre de plusieurs dizaines de milliers de travailleurs sous contrat dans les usines d'armement ou pour le terrassement des tranchées mais dont la plupart, à l'exception de deux à trois milles sont repartis en Chine à la fin du conflit. Or, c'est précisément des matériaux concernant ces Chinois-là, et avec lesquels pourtant la population contemporaine a, dans sa majorité, peu de liens directs, qui devaient constituer une part importante de ce musée. Cela illustre bien comment des filiations entre populations différentes peuvent être fabriquées. Sur un autre plan on peut noter la multiplication de travaux historiques sur les migrations chinoises notamment de la part d'universitaires d'Asie du sud-est ou d'Amérique du nord, d'origine chinoise directe ou à une ou plusieurs générations. Il s'agit véritablement d'entreprises visant à créer une histoire commune "autorisée » (la majorité des acteurs sont des universitaires) propice à exprimer une dimension commune des origines. Mémoires savantes, mémoires individuelles, mémoires du quotidien et des événements visent à refigurer et à présenter à l'entendement, à arranger les séries discontinues des expériences individuelles afin de les rendre intelligibles. Et à les inscrire dans un cours du temps qui permette de donner un sens à ces événements en les plaçant dans une expérience collective : elles permettent de leur donner une continuité. Cette somme d'expériences reconstituée comme expérience collective permet d'orienter et de réinscrire dans un sens plausible, le cours chaotique des expériences individuelles - des joies, des douleurs et des peines ${ }^{6}$ - qui sans cela resteraient morcelées. Cette histoire ainsi constituée est le mode narratif qui permet de décliner (de dire et de se dire) les aventures personnelles sans que celles-ci ne soient éparpillées en autant d'événements in-sensés. Elle les arrange en un tout dont la cohérence est donnée par la possibilité pour chacun de s'inscrire dans un cours, une suite, une continuité entre ceux qui sont morts, ceux qui sont encore et ceux qui seront. Ce tout, cette mémoire/histoire collective, constitue le socle des représentations des individus dans un passé désormais commun et des projections de ces mêmes individus dans le futur. La continuité inflexible du temps dont l'horizon anthropologique est la mort individuelle est alors humanisée à travers une généalogie de soi dans les prédécesseurs et une reproduction de soi dans les successeurs. Le temps ainsi maîtrisé permet de saisir l'espace, la dispersion : la diaspora. Il offre comme une continuité aux espaces disjoints que sont les différentes localisations de la diaspora : il donne à penser la diaspora comme une unité spatiale et temporelle, comme un êtreensemble unitaire alors qu'elle est, aussi, dispersion géographique des corps et des mémoires individuelles. Ce mouvement de ressaisissement de la diaspora par elle- 
même, comme pour conjurer sa division physique, à travers diverses procédures représentatives est bien montré par Alain Médam ${ }^{7}$ notamment dans "Mondes juifs, l'envers et l'endroit » dont le titre aurait pu être, selon l'auteur : « Le monde est dans le lieu, le lieu est dans le monde ", titre qui aurait mieux suggéré ce ressaisissement et cette fulgurance scalaire où le local saisit le global en même temps qu'il est saisi par lui. Cette métaphore qui est rapprochée de celle de l'hologramme est probablement la plus appropriée pour figurer les effets de la fameuse « globalisation ». Qu'elle soit aussi celle qui rende bien compte du paradoxe diasporique suggère fortement que les deux phénomènes ont des apparentements puissants. Le développement de relations interpolaires entre les différents lieux d'implantation, le renforcement de la multipolarisation de la migration par de nouveaux points de fixation et la densification de l'espace migratoire par des flux migratoires constants permet l'émergence d'une conscience diasporique. Elle donne lieu, en même temps qu'elle en est le résultat, à la constitution d'une mémoire/histoire collective qui prend le pas sur celle attachée au lieu d'origine: la Chine plus ou moins mythique selon qu'on en est directement en provenance ou que l'on soit descendant de migrants installés depuis plusieurs générations en Asie du sud-est. Cette mémoire/histoire déplace le lieu mythique d'origine vers un espace métaphorique qui serait celui de la diaspora, multitude de lieux où se trouvent les autres semblables. Et la fidélité n'est plus tant celle au lieu d'origine qu'à ce non-lieu matériellement inconsistant. Alors la question des origines, laquelle combine comme nous l'avons vu origine géographique et généalogie, ne s'envisage plus seulement comme une linéarité, une continuité temporelle reliant les contemporains à un point mythique d'origine, directement ou à travers les prédécesseurs, mais dans une contiguïté des lieux donnant espace et origine à ces contemporains: comme une monade topographique où les "ancêtres" seraient partout $^{8}$. De sorte que cet espace, ce non-lieu de la diaspora, serait leur séjour, ils y seraient virtuellement présents. Cette perte progressive de la référence au lieu mythique d'origine (la matrice originelle) au profit d'une référence à une monade extra-territorialisée est un changement majeur dans le système de représentation de soi d'un groupe ethnique.

Contiguïté géographique Cette généalogie par la continuité qu'elle instaure entre des individus situés dans des pays différents en leur offrant une origine commune sert de base à la formation d'une identité ethnique transnationale. Cette continuité généalogique d'autre part argumente une contiguïté géographique en localisant l'origine du groupe désormais défini par cette identité, non plus dans le pays d'origine mais dans une monade géographique constituée par les différents points d'ancrage de cette diaspora. Cette vision de soi dans l'espace que se propose la diaspora permet alors de transformer la dispersion en ressources spatiales, elle n'est plus pensée comme handicapante (n'être pas réunis en un lieu, en une matrice unique) mais comme un moyen positif de reproduction. Ce processus permet également de penser l'unité de la dispersion. Il consiste à traduire du temps en espace en transformant de la continuité généalogique en contiguïté géographique. La fabrication d'une identité diasporique à travers l'invention d'une généalogie commune permet en effet de réunir par l'esprit les lieux de la dispersion en une matrice d'origine qui offre alors un espace d'origine, non pas aux ancêtres, aux prédécesseurs (cet espace mythique on le connaît, c'est, dans notre exemple, la Chine) mais aux contemporains. Cet espace, il faut y insister, n'est plus seulement celui des ancêtres ou des prédécesseurs mais l'ensemble des lieux de présence de la diaspora qui ne sont plus pensés comme dispersés puisque réunis dans le 
geste généalogique. Ces lieux sont, à la limite, l'ensemble du globe terrestre habité. La diaspora, lorsqu'elle est pensée comme telle par les sujets qui la constituent, est alors une humanisation de la terre - et non plus d'un territoire - puisque celle-ci se présente dès lors dans sa totalité comme un réceptacle qui donne origine et avenir à une multiplicité dispersée - au détriment d'un lieu commun propre à chacun des groupes humains (le pays où l'individu sédentaire naît, vit et meurt). L'invention d'une continuité généalogique le long de la dimension du temps (l'invention d'un temps donc) permet la fabrication d'une contiguïté géographique (l'invention d'un espace). La réponse à la question des origines (question qui fonde l'identité ethnique) est recherchée de deux manières : selon une ligne généalogique (de qui provenons-nous ?) et selon une ligne géographique (d'où provenons-nous ?). Ce qui est recherché est donc la matrice où s'abolissent le temps et l'espace puisqu'elle en est le commencement. Cette figure est mobilisée par les sujets pour s'expliquer leur origine (de qui et d'où provenons-nous?) et définir leur identité (qui sommes-nous ?). Mais concernant les populations en diaspora (et c'est tout l'intérêt de leur étude en tant que cas limite) dont les lieux d'origine peuvent être très divers ${ }^{9}$, il y a une difficulté logique à penser une même matrice. Il faut certes avoir recours à la généalogie, mais les généalogies « réelles» proposent des origines diverses (par exemple, multitude des origines nationales pour les Chinois) qui de surcroit se compliquent lorsqu'il y a des mariages mixtes (inter-ethniques). De sorte que par une sorte de forçage logique, la matrice en vient à être non pas un lieu unique (celui-ci demeure toutefois mais sous une forme mythique) mais une multitude de lieux. Mais en même temps, cette multiplicité est réunie par le geste généalogique. Et c'est la dispersion même qui fait matrice (géographique). C'est le déploiement spatial du corps social, jusqu'à la limite extrême de l'ensemble du globe qui s'offre comme lieu géographique d'origine. C'est en ce sens que l'on peut dire que la continuité généalogique fabrique de la contiguïté géographique.

Extra-territorialité La construction d'une mémoire/histoire collective permet de réunir les lieux de la diaspora en un territoire virtuel, de regrouper par la pensée les lieux en un ensemble, en un espace métaphorique. Ce mouvement pour penser la diaspora dans l'espace participe d'un processus d'extra-territorialisation. De façon très succincte ${ }^{10}$ on peut dire que dans ce processus il y a d'abord une prise de conscience de la configuration spatiale de la diaspora (multipolarité de la migration que les individus, en plus de ce qu'ils savent à partir des médias communautaires - informations sur les populations chinoises dans diverses parties du monde - peuvent concrètement percevoir dans la variété des origines nationales des autres Chinois qu'ils côtoient ${ }^{11}$, interpolarité des relations à travers les liens réels entretenus avec d'autres personnes dans différents pays) puis une positivation de cette configuration à travers le développement d'une culture de la diaspora par la mise en relief de références et de valeurs transnationales. L'existence de relations interpolaires sur une base identitaire (relations avec des semblables) indiquent que l'on n'est pas dans une situation de disparition ou de dilution de ces groupes dans les sociétés d'accueil. Une identité s'est construite qui se superpose aux autres identités que ces groupes peuvent avoir ; elle est le support de ces relations en même temps qu'elle en est le produit, et son caractère décisif est d'être à la fois identitaire ethnique (ce sont des mêmes - dimension identitaire - parce qu'ils pensent partager la même origine - dimension ethnique) et transnationale. Et il faut entendre maintenant identité ethnique transnationale dans deux sens au moins : une identité qui transcende (a) les frontières stato-nationales des 
pays où sont localisés les Chinois de la diaspora (b) les différentes appartenances nationales des Chinois de la diaspora.

6 Peut-on parler de territoire à propos d'une diaspora, d'un corps social défini par sa dispersion? L'utilisation de l'idée d'extra-territorialité traduit la difficulté qu'il y a à traiter de cette question mais elle a l'avantage de permettre d'interroger les conceptions classiques du territoire. Quel serait, où serait le lieu de la diaspora? Comment penser l'unité d'un corps dispersé ? L'idée développée est que ce lieu est un non-lieu, une a-topie. Le sentiment d'extra-territorialité est ce qui configure la représentation dans l'espace que la diaspora se fait d'elle-même. Mais c'est à l'évidence une extra-territorialité vis à vis des territoires nationaux. L'on retrouve ainsi la prégnance du modèle national-territorial qui lui-même s'appuie sur une idée du territoire comme espace circonscrit par la présence pérenne d'une population. Le nonlieu de la diaspora est également une utopie (qui trouve sa réalité dans le sentiment d'extra-territorialité) dans la mesure où elle affirme la possibilité de vivre au-dessus (échelle transnationale) ou en dessous (échelle locale) du niveau national, c'est à dire au-delà ou en deçà des cadres qui dominent absolument les représentations et l'organisation sociale de l'espace: c'est en faisant sauter le verrou national que la diaspora peut penser son unité. Le sentiment d'extra-territorialité permet donc de penser l'unité d'un corps dispersé en ouvrant le verrou national.

7 Parler d'extra-territorialité permet aussi de se démarquer de la conception du territoire qui l'envisage comme un espace continu circonscrit par la présence pérenne d'une population. L'on peut ainsi souligner que le rapport à l'espace de la diaspora est fondamentalement différent et faire ressortir le caractère spécifique de ce rapport dans lequel espace/lieu d'origine et espace/lieu de vie sont disjoints dans la réalité mais en même temps constamment reliés dans l'esprit par le fait même d'appeler cet espace d'origine à travers des évocations. Comme s'il fallait constamment invoquer la matrice d'origine (le passé) pour situer le lieu où l'on vit présentement. Dans le cas des diasporas, notamment dès lors qu'elles comptent plusieurs générations, il y a comme on l'a vu plus haut une difficulté - que l'on qualifierait dans le langage contemporain de cognitive - à se représenter un espace commun en ceci que le lieu d'origine d'un membre du groupe peut être différent de celui de nombreux autres membres de ce groupe. De sorte qu'établir une origine commune présente une sorte d'impossibilité logique du type : on ne peut pas être à deux endroits à la fois, et donc on ne peut pas être de deux endroits à la fois. Il faut alors avoir recours à une filiation, une généalogie afin de situer cette origine (mes parents, mes grands-parents sont nés en tel lieu/ viennent de tel lieu...) et surtout oblitérer le lieu réel de leur apparition (leur lieu de naissance). Mais cette position n'est pas tenable puisque ce lieu d'apparition de l'individu existe et est présent à son esprit. Il lui faut alors tenir ensemble ces deux lieux disjoints. Et il ne peut le faire qu'en invoquant une généalogie. Comme tout migrant il est ici et là-bas, mais ce là-bas n'est pas à proprement parler le sien puisqu'il n'y est pas né, il est celui de ces ascendants. C'est ce rapport particulier à l'espace qu'entretient l'individu diasporique que nous pouvons traduire par l'idée d'extraterritorialité. Il y a comme un impératif d'ubiquité dans la façon de se penser dans l'espace, il lui faut, comme on l'a dit, faire cette double opération de se situer dans deux lieux différents. Mais il faut plus dès lors que l'on se relie au moins par l'esprit à d'autres individus avec lesquels on pense partager la même origine : il faut se situer avec ceux-là qui ont, eux aussi, cette double origine et dont, si une est la même que la nôtre, l'autre est différente, de sorte que par un autre processus je me situe ailleurs 
parce qu'ils ont au moins une même origine que la mienne. Par l'effet de ce triangle (lui et moi qui sommes en partie des mêmes et eux, les prédécesseurs à qui nous devons à la fois notre identité et notre différence), les lieux sont rassemblés le long de la ligne du temps, reliés en un espace qui peut s'envisager alors comme un espace commun. Mais l'expérience quotidienne ramène à l'évidence que cet espace est discontinu. Il n'est commun que par une opération de l'esprit, et non parce que l'on est en proximité physique puisque tel n'est pas le cas. C'est un territoire virtuel. Le terme d'extraterritorialité semble bien rendre compte de cette situation. Mobilisation des ressources spatiales Les conditions qui rendent possible la transformation de la dispersion en ressource spatiale sont l'amélioration des moyens matériels de circulation des personnes et des valeurs matérielles et immatérielles (marchandises, informations, codes, normes, modèles culturels...) qui permettent d'activer les relations interpolaires, le développement d'une identité ethnique transnationale par la création d'une mémoire/ histoire diasporique, la création d'un nouveau rapport au territoire par la transformation d'une continuité généalogique en une contiguïté géographique qui donne naissance à un sentiment d'extra territorialité. Le tout permettant de penser la configuration morphologique de la diaspora comme disposition positive. Ces processus rendent possible une optimisation des ressources spatiales de la diaspora que représentent la multipolarité de la migration et l'interpolarité des relations, ce qui, en retour, renforce le caractère diasporique. La notion de ressources spatiales permet de comprendre comment la configuration diasporique peut être utilisée positivement par les acteurs. Cette mobilisation trouve son origine dans le fait de savoir qu'existe la possibilité de la mobilité du fait de la présence ailleurs d'autres semblables, d'où l'importance de la dimension identitaire. Ces ressources spatiales sont utilisées dans différents domaines et mobilisées à différentes échelles.

8 L'exemple classique est la mise en place de réseaux commerciaux entre la Chine et les différents pays d'implantation de la diaspora, ces réseaux se sont considérablement renforcés depuis l'ouverture économique de la Chine. A une échelle plus fine cela se traduit par une extension des réseaux de distribution dans différents points de fixation dans un pays donné au fur et à mesure que la migration s'y étend. Plus intéressant du point de vue qui nous intéresse ici est l'établissement de réseaux commerciaux entre les différents lieux de fixation. La configuration diasporique permet une diversification des aires géographiques d'approvisionnement et de distribution. Dans les commerces d'alimentation, pour prendre un cas aisément accessible, on peut remarquer que l'origine des produits spécialisés concerne plusieurs dizaines de pays différents qui, outre la Chine continentale, vont de Taiwan, Singapour ou Hong Kong à Hawaï en passant par la Thaïlande, le Brésil ou encore l'Espagne et la Californie. Un relevé réalisé en 1998 dans un supermarché asiatique de Paris a permis de comptabiliser 37 pays d'origine des produits. Tous ces pays enregistrent la présence de Chinois d'outre-mer. Autre exemple, une bonne partie des produits africains distribués en France par des commerces chinois spécialisés orientés vers les populations subsahariennes sont collectés dans différents pays d'Afrique par d'autres commerçants de la diaspora installés dans ces pays (poisson séché, tubercules, épices...). Des réseaux de même nature mais de moindre extension géographique se sont mis en place pour des produits non alimentaires: produits de type artisanat asiatique, meubles, vaisselle, objets décoratifs etc. souvent en provenance d'Asie du sud-est. Les grossistes maroquiniers chinois de Paris quant à eux s'approvisionnent par exemple pour partie depuis une dizaine d'années en Italie auprès de compatriotes établis dans la région de Florence. 
Dans le domaine de la confection, des échanges sont signalés entre la France, l'Italie et l'Espagne.

9 A une autre échelle, les ressources spatiales peuvent être mobilisées pour le déplacement des affaires d'un pays à l'autre lorsque les conditions d'exploitation se détériorent. Ainsi s'expliquent le repli dans les années 60 et 70 des capitaux d'Indonésie vers Singapour et la Thaïlande notamment. De même manière s'explique aujourd'hui le déplacement de capitaux depuis l'Asie du sud-est vers la côte ouest américaine afin de profiter d'opportunités économiques (flux financiers en provenance de Taiwan ou de Singapour) et/ou pour anticiper des difficultés (flux originaires de Hong Kong en rapport avec sa rétrocession à la Chine continentale). Jan Lin souligne l'importance de la présence d'une chinatown pour attirer les investisseurs ${ }^{12}$.

10 C'est sur le plan de la migration que les ressources spatiales ont le plus d'effets, directs et indirects, dans la mesure où la circulation des personnes et leur installation dans d'autres pays accroissent le potentiel de circulation des valeurs matérielles et immatérielles puisque ce sont ces individus qui les mettent en circulation. Cela se traduit par exemple par une réorientation des flux migratoires en fonction des conjonctures locales ou nationales. C'est ainsi qu'en France, les réfugiés d'Asie du sudest d'origine chinoise accueillis durant les années soixante-dix et quatre-vingt dans des centres d'hébergement pour réfugiés en province ont rapidement rejoint des compatriotes déjà installés à Paris. En Grande Bretagne la même situation s'est présentée ainsi que le montre Vaughan Robinson ${ }^{13}$. En Italie, la présence de Chinois originaires de la province du Zhejiang, notamment à Florence, a permis à des compatriotes en situation irrégulière à Paris d'immigrer dans ce pays et d'y régulariser leur présence comme le montrent Giovanna Campani et Lucia Maddi ${ }^{14}$. Le même phénomène s'est produit en Espagne dans les années 1991-1992 ${ }^{15}$ ou encore à une époque différente, l'entre-deux-guerres, pour le même groupe mais cette fois-ci entre Paris et Amsterdam ${ }^{16}$. L'adaptabilité de leurs réseaux migratoires aux variations des politiques migratoires des Etats-nations est une caractéristique commune à un grand nombre de groupes migrants qu'ils soient maghrébins, africains, indiens etc. leur extension cependant, et leur densité offrent de plus grandes opportunités de migrer et favorisent davantage les circulations de personnes. L'actualité nous offre un exemple tragique de mobilisation des ressources spatiales. En Indonésie où l'on compte de 7 à 9 millions d'individus d'origine chinoise (immigrés ou descendants d'immigrés à une ou plusieurs générations), les manifestations contre le gouvernement et le président Suharto ont parfois dégénéré en émeutes contre les Chinois ou identifiés comme tels. Durant le premier semestre de 1998, mille deux cents d'entre eux auraient été assassinés par les émeutiers dans la seule ville de Jakarta ${ }^{17}$, de nombreux commerces et résidences leur appartenant incendiés ${ }^{18}$. D'après le Straits Times de Singapour daté du 18 avril 99, de 100000 à 125000 personnes d'origine chinoise auraient quitté l'Indonésie en un an principalement vers Singapour, la Malaisie, l'Australie ou encore Hong Kong où l'on comptait en février 1999, 38300 indonésiens d'origine chinoise (AFP, Hong Kong, 21 mai 1999). Des flux plus modestes se sont dirigés vers la Thaïlande et les Etats-Unis et d'autres destinations où l'on enregistrait la présence de Chinois d'outremer. Parallèlement, ces manifestations ont provoqué le départ de capitaux importants ${ }^{19}$ vers les mêmes destinations attestant par-là l'efficacité des réseaux financiers de la diaspora en Asie du sud-est. De nombreux autres exemples de mobilisation des ressources spatiales pourraient être exposés, ils montreraient que la multipolarité de la migration, l'interpolarité des relations et l'extra-territorialité jouent un rôle essentiel 
dans le fonctionnement concret de la diaspora et qu'elles sont constitutives de celle-ci. À cela il faut ajouter le rôle décisif qu'a pu jouer la diaspora chinoise dans le développement de certains pays où ses effets semblent considérables parmi les "dragons» de l'Asie (Singapour, Hong Kong, Taiwan) et les "petits dragons » ou «bébés tigres " (Thaïlande, Malaisie...) ainsi qu'en Indonésie et aux Philippines. Mais aussi dans celui de la Chine continentale ${ }^{20}$. Ainsi du fulgurant développement économique de certaines provinces chinoises (Fujian et Guandong) qui doit surtout aux investissements étrangers, c'est à dire en l'occurrence des Chinois d'outre-mer. S'agit-il seulement du fait d'une diaspora "riche ", puisque c'est souvent ainsi qu'est présentée la diaspora chinoise, ou plutôt le résultat d'une efficacité liée à sa situation précisément de diaspora? De la fluidité que donne la configuration diasporique et qui permet des prises de richesses çà et là et leur placement et déplacement judicieux ?

Le cas de la diaspora chinoise peut être utilisé à titre de comparaison dans l'étude des réseaux transnationaux entre l'Europe et le Maghreb. Mais il est certain que les Chinois d'outre-mer et ceux qu'on pourrait appeler les « Maghrébins d'outre-mer " présentent un certain nombre de différences dont une des plus significatives est peut-être le temps, la durée de la dispersion : les Chinois d'outre-mer ont commencé à émigrer de manière significative durant la deuxième moitié du siècle dernier alors que les Maghrébins l'ont fait dans les années soixante. Néanmoins pour ces derniers le temps commence à compter et il y a maintenant deux (ou plus) générations nées "outremer ». Les lignes précédentes suggèrent l'idée que le développement économique des nouveaux dragons tels que la Malaisie, la Thaïlande, et maintenant le Vietnam a été possible du fait de l'existence réseaux économiques ethniques (principalement chinois pour des raisons historiques). Le cas des dragons plus anciens - Taiwan, Hong Kong, Singapour - est un peu particulier du fait qu'il s'agit de places dont la très grande majorité de la population est d'origine chinoise. Il est à noter que ces réseaux se sont constitués à l'initiative d'individus issus d'une main d'œuvre chinoise ayant migré dans différents pays de l'Asie du sud-est à l'occasion de la mise en valeur de cette région par les puissances coloniales. Cette caractéristique - entreprises et réseaux économiques créés par des «immigrés » - devrait permettre la comparaison avec ceux créés par d'autres populations migrantes telles que les Marocains ou les Tunisiens ${ }^{21}$ ou encore les Mourides $^{22}$ et bien d'autres encore qui construisent eux aussi des dispositifs économiques internationaux.

\section{NOTES}

1. Sur les Chinois d'outre-mer voir notamment Trolliet P., La diaspora chinoise, Paris, Presses Universitaires de France, Que sais-je ? n 2879.

2. Sont repris ici sous une forme synthétique plusieurs éléments développés dans d'autres textes, par exemple : Ma Mung E., « Non lieu et utopie, la diaspora chinoise et le territoire ", L'Espace géographique, vol. 23, n² 2, 1994, pp. 106-113, repris dans une version plus détaillée in Bruneau M. (dir.), Diasporas, Montpellier, Reclus, 1995, pp. 163-173. Ma Mung E. «Intégration locale et territoire global », Cultures en mouvement, 
$\mathrm{n}^{\circ}$ 10, 1990. Ma Mung E. (dir.), Mobilités et investissements des émigrés, Maroc, Tunisie, Turquie, Sénégal, Paris, L’Harmattan, 1995, 270 p.

3. Ma Mung E., op. cit., 1994 et 1995.

4. Les Chinois d'outre-mer adoptent très souvent la nationalité du pays d'accueil (cf. P. Trolliet, op. cit.). Si on retient ce caractère comme indice d'intégration ils sont le plus souvent "intégrés" aux pays dans lesquels ils séjournent.

5. L'identité ethnique est prise ici au sens weberien de croyance partagée en une même origine, c'est ce qui la différencie des autres formes d'identité sociale (professionnelle, religieuse, politique...).

6. Ceci est d'autant plus important qu'une bonne partie de la population chinoise en France est constituée de réfugiés de l'Asie du sud-est ayant quitté leur pays d'origine dans des conditions dramatiques.

7. Médam Alain, Mondes juifs, l'envers et l'endroit, Paris, Presses Universitaires de France, 1991, $192 \mathrm{p}$.

8. Le rapatriement des corps vers « la terre des ancêtres » autrefois systématique (Jules Verne en tira même l'idée de son roman sur Les tribulations d'un chinois en Chine) devient moins fréquent.

9. En France on enregistre dans les populations d'origine chinoise plus d'une vingtaine d'origines nationales différentes, idem en Espagne aujourd'hui et probablement aussi en Grande Bretagne, et un nombre encore plus grand en Amérique du nord, près d'une trentaine à Montréal par exemple et une quarantaine aux Etats-Unis.

10. Voir E. Ma Mung, op. cit.

11. Voir note 10.

12. Lin J., « Flux de main d'œuvre et de capitaux chinois vers les Etats-Unis », Revue Européenne des Migrations Internationales, vol. 8, n 3, 1992, pp. 73-90. Waldinger R., Tseng Y., " Divergent Diasporas : the Chinese Communities of New York and Los Angeles Compared ", Revue Européenne des Migrations Internationales, vol. 8, n³, 1992, pp. 91-116.

13. Robinson V., " Une minorité invisible, les Chinois en Grande Bretagne », Revue Européenne des Migrations Internationales, vol. 8, n³, 1992, pp. 9-32.

14. Campani G., Maddi L., « Un monde à part : les Chinois en Toscane », Revue Européenne des Migrations Internationales, vol.8, n³, 1992, pp. 51-72. Voir également Campani G. et alii (éd.), L'immigrazionne silensioza, la communità cinesi in Italia, Milano, Edizioni della Fondazione G. Agnelli, 1994, 235 p.

15. Beltran Antolin J., «Immigrés chinois en Espagne ou citoyens européens ? », Revue Européenne des Migrations Internationales, vol. 13, n², 1997, pp. 63-79.

16. Franck P., « Immigration et entreprenariat, les Chinois aux Pays Bas », Revue Européenne des Migrations Internationales, vol. 8, n³, 1992, pp. 33-50.

17. D'autres émeutes anti-chinoises meurtrières ont eu lieu dans d'autres villes, notamment à Medan au nord de Sumatra.

18. Ces émeutes anti-chinoises dont le motif est la supposée mainmise des Chinois sur l'économie indonésienne, leur collusion avec les pouvoirs en place et donc leur supposée responsabilité dans la situation économique désastreuse de la population et du pays, s'inscrivent dans une longue suite d'événements dont les plus graves sont les manifestations anti-chinoises du milieu des années soixante qui se sont soldées par la mort de plusieurs dizaines de milliers de personnes. Ces pogroms se sont déroulés durant la période d'agitation précédent le putsch militaire du 30 septembre 1965 qui devait écarter Sukarno et amener Suharto au pouvoir en juillet 1966. 
19. Business Times, Singapour, du 15 avril 1999.

20. Trolliet P., op. cit. Lever-Tracy C. et alii, The Chinese Diaspora and Mainland China : An Emerging Economic Synergy, London, Mac Millan Press, New York, St Martin's Press, 1996, $310 \mathrm{p}$.

21. Voir Tarrius A., Arabes de France dans l'économie souterraine mondiale, Paris, Editions de l'Aube, 1995, 220 p. : Boubakri H., Les entrepreneurs migrants du sud-est tunisien, in Césari J. (dir.), Réseaux transnationaux entre l'Europe et le Maghreb, Rapport de recherche pour la Commission des Communautés Européennes, 1996, volume 1 pp. 17-29, volume 2 pp. 3-58 et pp. 192-212. Césari J., « Les réseaux transnationaux entre l'Europe et le Maghreb : l'international sans le territoire ", Revue Européenne des Migrations Internationales, vol. 13, n² 2, 1997, pp. 81-94.

22. Ebin V., Négociations et appropriations : les revendications des migrants sénégalais à New York, communications au colloque " Systèmes et dynamiques des migrations internationales ouest-africaines, Dakar, 3-6 décembre1996.

\section{INDEX}

Index géographique : Chine

Mots-clés : Diaspora, flux, migration, mobilité, Mondialisation

\section{AUTEUR}

\section{EMMANUEL MA MUNG}

Chargé de recherche au CNRS, équipe MIGRINTER de l'UMR 6588, CNRS/Universités de Poitiers et de Bordeaux. 